\title{
Detecting Activities from Body-Worn Accelerometers via Instance-based Algorithms
}

\author{
Nicola Bicocchi, Marco Mamei, Franco Zambonelli \\ Dipartimento di Scienze e Metodi dell'Ingegneria, University of Modena and Reggio \\ Emilia, Italy
}

\begin{abstract}
The automatic and unobtrusive identification of user's activities is one of the challenging goals of context-aware computing. This paper discusses and experimentally evaluates instance-based algorithms to infer user's activities on the basis of data acquired from body-worn accelerometer sensors. We show that instance-based algorithms can classify simple and specific activities with high accuracy. In addition, due to their low requirements, we show how they can be implemented on severely resource-constrained devices. Finally, we propose mechanisms to take advantage of the temporal dimension of the signal, and to identify novel activities at run time.
\end{abstract}

Key words: Context-aware Computing, Body-worn Sensors, Motion Classification.

\section{Introduction}

The automatic and unobtrusive identification of user's activities from sensor data is one of the key and most challenging goals of context-aware computing [1]. Several opportunities can arise from the availability of such an information. For example, a service would be able to flexibly adapt its behavior to different circumstances (e.g., a smart phone application could turn silent, once recognizing that the user is in a theater watching a movie). As another example, the simple description of user's activities could automatically produce entries in blogs, diaries and social network sites [2].

While advances in hardware technologies (e.g., smart phones, wireless sensors and RFID tags [3]) are making feasible to collect a vast amount 
of information about the user in an unobtrusive way, it is still difficult to organize and aggregate all the collected information in a coherent, expressive and semantically-rich representation. In other words there is a gap between low-level sensor readings and their high-level context description [4].

In this paper we tackle the problem of turning low-level accelerometer data acquired from body-worn sensors into high-level activity descriptions. Body-worn accelerometers are suitable for this kind of applications: they are small and economic, could be easily integrated in everyday objects and clothes, and they are already integrated in a number of smart phones.

To efficiently classify human activities on the basis of accelerometer data we focus on instance based mechanisms. Instance-based classifiers do not build an explicit, declarative representation of the class of interest but rely on the class labels attached to the training samples similar to the test sample to be classified. These methods have thus been called lazy learners, since "they defer the decision on how to generalize beyond the training data until each new query instance is encountered" [5]. We focused on these algorithms in that they are easy to implement, they offer state-of-the-art classification performance, the classification procedure can be easily understood (it does not rely on black-box models like in neural networks), they support on-line classification and training and, as detailed in the following sections, they can be implemented on resource-constrained devices.

Although the problem of classifying user activities on the basis of bodyworn accelerometers has already been investigated in several other researches $[6,7,8,9]$ (see Section 2 for details), the contribution of this paper is to show that simple instance-based algorithms can classify accelerometer data more accurately than several other approaches employing more complex algorithms. In addition, we show that instance-based algorithms can be executed on resource constrained devices like sensor motes.

More in detail, the paper presents the following contributions and insights:

1. We show that simple instance-based (IB) algorithms offer state-of-theart classification performance in this scenario (80\% classification precision and recall with 16 classes). In addition, we verified that they can be easily implemented on resource constrained sensors. In particular, the mainstream argument that IB consumes too much memory because it stores individual feature vectors (see later) is unfounded. In this scenario, IB can be implemented on memory-constrained devices keeping state-of-the-art performance. 
2. We highlight that body-worn accelerometers are not only useful to recognize simple motion/ambulation states (e.g., walking, running) like in most of the related work. These sensors can also recognize and discriminate among specific activities in other scenarios (e.g., use a PC, stir pasta, drive the car). This supports the use of accelerometer data and IB-algorithms to fulfill the context-awareness vision.

3. We present a mechanism allowing IB classification to take into consideration the time series of the acceleration data and to aggregate classification results over a given time frame to considerably improve classification precision and recall.

4. We present a mechanism to introduce and identify new activity classes at run time. One of the main problems of IB classifiers is that they classify any new pattern as one of the predefined classes, no matter how different the new pattern is from the ones in the training set. Instead, practical systems require a mechanism to understand that a pattern deviating from the ones seen previously could represent a completely new activity rather than a different "gait" for the predefined ones.

Accordingly, the remainder of the paper is organized as follows: Section 2 surveys related work in this area. Section 3 presents the instance-based algorithms we used to classify activities using acceleration data and illustrates the possible applications enabled by these algorithms. Section 4 presents some novel mechanisms we introduced to better deal with the sensors' time series and to enable the on-line discovery of new classes. Section 5 describes the collection of the dataset. Section 6 presents the classification performances of our proposal. Section 7 details some results illustrating the suitability of the proposed mechanism to resource-constrained sensors. Section 8 concludes.

\section{Related work}

The problem of recognizing user's activities on the basis of accelerometer data has been studied in a large number of works. Basically, all the approaches share the same basic framework:

1. A number of accelerometers are worn by the user typically at specific positions on her body.

2. Data is collected on a device (smart phone, laptop) and the user is asked to label his/her activities to provide ground truth information. 
3. Feature vectors are extracted from the raw accelerometer data.

4. A pattern recognition algorithm (whether instance-based or using other mechanisms, such as Hidden Markov Model and Support Vector Machine) is used to learn a model to recognize user's activities on the basis of the feature vectors.

5. Once this training phase is concluded, new data is collected while the user performs daily activities. Such data is converted in feature vectors and then classified.

6. Some standard measures (e.g., accuracy, confusion matrices) are used to evaluate classification performances.

Since the basic framework of all the approaches in the area is almost identical, we can present their setting and results in a single table (see Figure 2 ). For each of the related works being surveyed, we describe the number of accelerometers being used, where they have been located, the number and kind of activities the system tries to recognize, the classification mechanism being used, and the resulting classification accuracy.

Looking at the table, it is possible to see that apart from a few proposals $[10,6]$ most of the literature recognize a rather limited set of ambulatory activities. It is also worth emphasizing that in [10] 5 accelerometers are used instead of 3. This can obviously provide more data and ease the classification process. On the other side, [6] exhibits large variance in classification precision and recall. Despite a high number of activities is considered, some of them are poorly recognized (less than $4 \%$ accuracy).

Although this table is useful to present several related coherently works, it does not allow for simply comparing the reported accuracies and deduce that one work is better than another. These works are applied to different datasets, thus it is not correct to compare their results: it can be more difficult to classify 2 similar activities than to separate 20 "disjunct" ones. Similarly, one work can capture several expressions of the same activity (e.g., different ways of running), while another one might only capture only a few. Despite these limitations, the table gives an overall view on the classification task being addressed by the related work and on the obtained accuracy.

Our approach demonstrates that simple IB algorithms can reliably recognize a fairly large number of (also not ambulatory) activities.

We want also to emphasize that the use of IB approaches has recently gained a lot of attention in research and applications in several other areas. 
One of the best known concrete applications developed with IB mechanisms is Google's "Did you mean?" tool. It corrects errors in search queries, not through a complex parsing of the query but by memorizing billions of query-answer pairs and suggesting the one closest to the users query.

In [11] a system to classify images on the basis of IB algorithms is presented. The system uses a large dataset of 80 millions images collected from the Internet and labeled with one of the English nouns in the WordNet lexical database. Using IB approaches, new images are classified on the basis of the closest images in the dataset. This work demonstrates that IB algorithms obtain state-of-the-art performances also on complex computer vision tasks.

The work presented in [12] applies IB algorithms to bio-informatics. It compares different classification algorithms over typical bio-informatics datasets. IB algorithms consistently rank at the top of that list. Similarly, for textclassification, [13] shows that IB algorithms outperforms most of the (more complex) mechanisms for text classification.

These results further motivate us in experimenting IB algorithms in the area of human activity classification.

\section{Instance-Based Classification of Human Activities}

Instance-based classification algorithms can be generally divided into two broad categories: (i) approaches based on the distance between the new sample and the closest labeled instances, (ii) approaches based on the local density of labeled instances in the area close to the new sample.

In our work we experimented with 4 instance-based classification algorithms: K Nearest Neighbors (kNN, or IBk) represents the former category. Direct Density (DD), Class Local Outlier Factor (CLOF), Local Classification Factor (LCF) represent the latter one.

Density-based approaches have been used also because they provide effective techniques to detect outliers among the samples. As described in the next section, this characteristic is useful to introduce and identify new activity classes at run time.

\subsection{Feature Vectors Extraction}

Before describing the different instance-based classification algorithms, it is important to describe how feature vectors have been extracted from the accelerometer data. 


\begin{tabular}{|c|c|c|c|c|c|c|c|}
\hline Ref & N. Accel. & $\begin{array}{l}\text { Accel. } \\
\text { placement }\end{array}$ & $\begin{array}{l}\text { N. Activi- } \\
\text { ties }\end{array}$ & $\begin{array}{l}\text { Kind of } \\
\text { Activities }\end{array}$ & $\begin{array}{l}\text { Classification } \\
\text { Mecha- } \\
\text { nisms }\end{array}$ & Accuracy & Notes \\
\hline$[14]$ & $12 \mathrm{D}$ & thigh & 7 & ambulation & Rule-based & $93 \%-96 \%$ & (1) \\
\hline [15] & $23 \mathrm{D}$ & $\bar{L} / \mathrm{R}$ hip & 4 & $\begin{array}{l}\text { ambulation, } \\
\text { posture }\end{array}$ & PCA & $83 \%-90 \%$ & \\
\hline$[16]$ & $41 \mathrm{D}$ & $\begin{array}{l}\text { chest, } \\
\text { thigh, } \\
\text { wrist, } \\
\text { forearm }\end{array}$ & 9 & $\begin{array}{l}\text { ambulation, } \\
\text { posture, } \\
\text { typing, } \\
\text { talking, } \\
\text { cycling }\end{array}$ & $\mathrm{kNN}$ & $95.8 \%$ & \\
\hline$[17]$ & $21 \mathrm{D}$ & $\begin{array}{l}\text { chest, } \\
\text { thigh }\end{array}$ & 4 & $\begin{array}{l}\text { ambulation, } \\
\text { posture }\end{array}$ & Rule based & $89.3 \%$ & \\
\hline$[18]$ & $123 \mathrm{D}$ & $\begin{array}{l}\text { all major } \\
\text { joints }\end{array}$ & 6 & $\begin{array}{l}\text { ambulation, } \\
\text { typing, } \\
\text { writing, } \\
\text { shaking } \\
\text { hands }\end{array}$ & $\begin{array}{l}\text { Naive } \\
\text { Bayes }\end{array}$ & $65 \%-95 \%$ & \\
\hline [19] & $12 \mathrm{D}$ & wrist & 4 & $\begin{array}{l}\text { Kung-fu } \\
\text { movements }\end{array}$ & $\begin{array}{l}\text { Hierarchical } \\
\text { HMM }\end{array}$ & $96.7 \%$ & \\
\hline [20] & $12 \mathrm{D}$ & lower back & 6 & $\begin{array}{l}\text { ambulation, } \\
\text { posture }\end{array}$ & $\begin{array}{l}\text { Neural } \\
\text { Network }\end{array}$ & $85 \%-90 \%$ & \\
\hline [21] & $12 \mathrm{D}$ & knee & 6 & $\begin{array}{l}\text { ambulation, } \\
\text { posture, } \\
\text { cycling }\end{array}$ & SOM & $42 \%-96 \%$ & \\
\hline [10] & $52 \mathrm{D}$ & $\begin{array}{l}\text { hip, wrist, } \\
\text { arm, ankle, } \\
\text { thigh }\end{array}$ & 20 & $\begin{array}{l}\text { ambulation, } \\
\text { posture, } \\
\text { working, } \\
\text { home } \\
\text { activities }\end{array}$ & $\begin{array}{l}\text { Decision } \\
\text { Table, } \\
\text { IB, C4.5, } \\
\text { Naive } \\
\text { Bayes }\end{array}$ & $84 \%$ & \\
\hline [7] & $33 \mathrm{D}$ & $\begin{array}{l}\text { wrist, } \\
\text { waist, } \\
\text { shoulder }\end{array}$ & 8 & $\begin{array}{l}\text { ambulation, } \\
\text { posture, } \\
\text { brushing } \\
\text { teeth }\end{array}$ & HMM & $93.8 \%$ & $(2)$ \\
\hline [6] & $23 \mathrm{D}$ & hip, wrist & 34 & $\begin{array}{l}\text { ambulation, } \\
\text { posture, } \\
\text { working, } \\
\text { home act. }\end{array}$ & $\overline{\mathrm{LSA}}$ & $72.7 \%$ & (3) \\
\hline$[22]$ & $13 \mathrm{D}$ & waist & 8 & $\begin{array}{l}\text { ambulation, } \\
\text { posture, } \\
\text { brushing } \\
\text { teeth }\end{array}$ & $\begin{array}{l}\text { Decision } \\
\text { Table, } \\
\text { C4.5, kNN, } \\
\text { SVM, } \\
\text { Naive } \\
\text { Bayes }\end{array}$ & $73 \%-99 \%$ & (4) \\
\hline [23] & $13 \mathrm{D}$ & waist & 4 & $\begin{array}{l}\text { ambulation, } \\
\text { posture }\end{array}$ & $\begin{array}{l}\text { Gaussian } \\
\text { Mixture } \\
\end{array}$ & $85 \%$ & \\
\hline$[8]$ & $53 \mathrm{D}$ & $\begin{array}{l}\text { wrists, } \\
\text { arms, torso }\end{array}$ & 5 & $\begin{array}{l}\text { home, of- } \\
\text { fice act. }\end{array}$ & HMM & $90 \%$ & (5) \\
\hline $\begin{array}{l}\text { In this pa- } \\
\text { per }\end{array}$ & $33 \mathrm{D}$ & $\begin{array}{l}\text { arm, waist, } \\
\text { leg }\end{array}$ & 16 & $\begin{array}{l}\text { ambulation, } \\
\text { working, } \\
\text { home act. }\end{array}$ & IB & $95 \%$ & (6) \\
\hline
\end{tabular}

Figure 1: Related work dealing with activity recognition on the basis of accelerometer data. Notes: (1) Allows also dead reckoning, (2) Tested also on $13 D$ sensor whose position changes dynamically, getting 80\% accuracy, (3) Large variance in accuracy among activities (some activities are poorly recognized - less than 4\% accuracy), (4) Portability of the model across users, (5) Deals also with complex activities, (6) All activities are recognized with similar accuracy. Acronyms: PCA (Principal Components Analysis), kNN (k Nearest Neighbors), SOM (Self-Organizing Maps), IB (Instance Based mechanisms), HMM (Hidden Markov Model), LSA (Latent Semantic Analysis), SVM (Support Vector Machine). 
We started by just considering feature vectors consisting of 9 elements corresponding to the $x, y, z$ components of the acceleration measured by 3 body-worn sensors (positioned at right arm, waist and right leg) in a single sampling (sensors are sampled at $10 \mathrm{~Hz}$ ). Each feature vector has been classified independently. The distance among these vectors have been measured in terms of Euclidean distance.

It is worth noticing that our sampling rate is lower than that reported in other related works. For example, the CenceMe project [9] measured that $40 \mathrm{~Hz}$ is the minimum acceptable rate for effectively classifying activities from a single 3D accelerometer embedded in a mobile phone. In our case, the use of 3 well-positioned 3D accelerometers allows to classify effectively accelerometer data acquired at lower sampling rates.

In the following Section 4.1, we describe how we extended this vector to take into account the temporal dynamics of the collected data.

\subsection{Instance-based Classification}

In this section we briefly describe the instance-based classification algorithms we adopted in our experiments.

k Nearest Neighbors (kNN, or IBk). The k Nearest Neighbors algorithm classifies a new sample $p$ by selecting the $k$ training samples that are closest to $p$ under some distance function (in this work we used the Euclidean distance). Then, $p$ is given the most frequent label appearing in the selected $k$ training samples. In the simplest case $(k=1)$, a new sample is classified the same as the closest instance in the training set.

Direct Density (DD). If the samples in the training set TS are unevenly distributed (like in most real cases), the $\mathrm{kNN}$ algorithm can fail to correctly classify a given sample if it falls in a region having a high-density of samples of another class. To deal with this issue, the Direct Density approach (DD) takes into consideration the average distance between the sample to be classified and the k-nearest neighbors of a given class. The sample is classified with the class of k-nearest neighbors at the minimum distance.

Class Local Outlier Factor (CLOF). While the DD algorithm tries to determine to which extent a new sample is close to the clusters in the training sets associated with the different classes, the Class Local Outlier Factor (CLOF) algorithm measures how much the new sample is an outlier with respect to that clusters. It does so, by considering the ratio between the DD measure of the sample to be classified $(q)$ and the average DD measure 
of the training samples close to $q$. If the $q$ sample is within a cluster such ratio will be close to 1 . Otherwise it will be much higher.

Local Classification Factor (LCF). The Local Classification Factor (LCF) linearly combines DD and CLOF classification $(L C F=D D+w$. $C L O F)$, trying to get the best trade-off between the two. The parameter $w$, used in the linear combination, determines to which degree the outlier factor of a sample $q$ is relevant for its classification. A higher value for $w$ leads to more correctly classified objects in the sparser classes, at the expense of incorrectly classified objects in the denser classes.

In Section 6 we will present the results of applying such algorithms to human action classification.

\section{Refining the Classification}

In this section we present some valuable refinements to the classification process. The first contribution allows to improve classification accuracy by taking into account the time series of the acquired data. The second contribution allows to discover new classes of activities at run time.

\subsection{Dealing with Time}

While some classification algorithms, such as HMM [5], inherently deal with the temporal relationships among the acquired samples, instance-based algorithms do not have such a mechanism built in their conception.

Still, for human activities classification such kind of temporal relationships are important. In fact, while individual sensor readings can be ambiguous and noisy, the pattern of a whole time-window of readings can be much more recognizable. In addition, assuming that human activities last for a minimum amount of time, it is possible to output class labels at a much lower rate (e.g., 1Hz) than sensor readings (e.g. 10Hz) to save computational resources (see Section 7.1).

Accordingly, we developed 2 simple yet effective mechanisms to represent temporal relationships in instance-based mechanisms.

Embedding Temporal Information in the Samples. This mechanism consists of adding elements, encoding temporal aspects, to the feature vector. In particular, starting from the 9-components feature vector (3 3-axis accelerometers) described in Section 3.1, we added mean and variance of the 
acceleration data computed over a sliding window. Furthermore, we considered sliding windows of both 10 and 20 consecutive samples (thus 1 and 2 seconds, respectively). We computed mean and variance of the module of the acceleration at each sensor $(3+3=6$ new components $)$ and concatenated these new components to obtain the new feature vector. The temporal meaning is thus encoded in the comparison between the actual observation and the mean and standard deviation computed in the sliding window.

This is a simple representation of the pattern of acceleration data, however it allows us to infer whether the current sensor reading is in-line with the recent past readings or it is an outlier in that respect. As illustrated in the experimental section, this simple temporal representation is effective and improves classification accuracy (precision and recall) by more than $10 \%$.

It is important to emphasize that this technique is fully integrated in the instance based algorithm. Embedding temporal data in the samples, enables us to use the same classification mechanism without additional modifications.

Majority Voting over Temporal Window. This mechanism starts from the consideration that all human activities have a minimum duration. Accordingly, it is convenient to aggregate classification results over a sliding window and perform majority voting on the classifications of that window. For example, let us assume a time window of 30 seconds and that sensors sample raw data at $10 \mathrm{~Hz}$ and produce time-enriched feature vectors once a second (as described in the above section). At this stage, suppose that the system classified, over the 30 seconds window, the user behavior as "running" 23 times and "walking" in the remaining 7 times. Majority voting will let the system classify the activity as "running" for the whole time frame. Starting from the fact that it doesn't make sense to recognize an action during only few seconds we can consider the classification glitches as an error and ignore them. Due to this, as illustrated in the experimental section, we switched from a simple instance-based classifier to a "window-based" classifier. We divide the time series of classified instances in windows with a variable length. Each window will be associated with the most frequent label present inside that time window.

\subsection{Dealing with New Classes}

One of the main problems in classification is that the system classifies any new pattern as one of the predefined activities, no matter how distant the new pattern is from any other pattern in the training set. In other words, 
the system is able to deal with only a predefined set of classes. Instead, a practical system requires a mechanism to add new classes at run time. It is worth noting that this problem extends the idea of the NULL class (i.e., the class that contains all the instances - outliers - that cannot be neatly classified in any of the specified classes) [24]. If sensors register a pattern significantly different form the ones describing the predefined classes, the system should create a new class (possibly asking the user for a proper label) and classify the new sensor readings accordingly.

On the one hand, instance-based algorithms are suitable for dynamically adding classes at run time. Since they do not have an explicit training phase, but all the computation is deferred at classification time, it is easy to add a new class just by adding samples with the new class label. On the other hand, it is rather difficult to decide whether a new sample is an outlier of a previously existing class or it is the first sample of a new class.

The straightforward solution of creating a new class if the new sample is farther than a threshold $\mathrm{T}$ from all the previously labeled samples does not work well in practice, producing low classification accuracy.

Thus, we developed a novel solution to this problem. As discussed in Section 3.2, Local Classification Factor (LCF) is suitable as an outlier detector. After choosing the weight $w$ to be used, for every sample $q$ to be classified, it produces a numeric value $L C F_{i}(q)$ associated with each class $i$ found in the training set. We modified our classifier to associate to a special class called new every item which has $\min \left(L C F_{i}(q)\right)$ greater than a threshold th. In Section 6 we show that, by properly tuning parameters $k, w$ and th it is possible to discover new classes at run time rather effectively.

A similar approach, grounded on statistical significance tests, has been presented in $[24,25]$. In our future work we will try to combine this with our approach to improve the recognition of new classes.

\section{Dataset Collection}

To test our proposal we set up a 4-node body-worn sensor network to collect acceleration data. The network consists of: 3 Sunspot sensors (http://www.sunspotworld.com) provided with 3D accelerometers attached to the right arm, waist and right leg of the person, and a back-packed UltraMobile PC (UMPC): Asus EeePC 701SD (http://eeepc.asus.com). The 3 Sunspots sample acceleration data at $10 \mathrm{~Hz}$ and transmit it to the ultramobile PC where data is classified on line on the basis of an instance-based 
algorithm (see Fig. 2). This simple set-up has two main advantages:

1. Simplicity. We developed and debugged classification algorithms on the ultra-mobile $\mathrm{PC}$, avoiding the complexity in programming with the sensors cumbersome libraries. This complies with the scientific aim of our work: we are not trying to create a ready-to-market solution, but to test models and algorithms. Moreover, high-level profiling tools to monitor the application memory budget and CPU performance can be readily used on the ultra-mobile $\mathrm{PC}$. Thus, we can conduct rigorous measurements to test whether the proposed approach can fit the resource constraints of the sensors.

2. Repeatability. Although the ultra-mobile PC classifies samples online, all the samples are also saved in files. This allows us to repeat experiments and re-verify our conclusions. Some of our datasets are available on line: http://pervasive2.morselli.unimo.it/ nicola/humanActions/

On the basis of our set-up, we performed two data acquisition campaigns:

1. Activities Dataset. To create this dataset we asked a group of 6 people to wear our sensor network and perform a series of 16 predefined activities: stir pasta (while cooking); climb stairs; sleep (lie in bed); run; use pc (type and move the mouse); wash hands; use playstation 3 (sat with the joypad in hand); read sat on the sofa; take the elevator; ride a bike; stand still; drive the car; eat pasta; drink at the bar (drink standing still); walk; dance (step feet and move hands rhythmically). These activities represent a mix of simple ambulatory activities and of specific actions dealing with house- or office-works. Collected sensor data has been clearly labeled with the associated activity.

2. Real-life Dataset. To create this dataset, 2 users (among the authors) wear the sensor network for 1 day, as they went about their normal lives. Ground-truth labels have been acquired with a separate recording application roughly synchronized with the sensor clock. Activities being annotated with the recording application are: chat being sat; drink standing; set up the table; clean kitchen's racks; eat; drive the car; clean the table; chat standing; work at the pc; read on the sofa; wash hands, take something from fridge

While the former dataset has all the sensor data labeled with the corresponding activities, the latter dataset has a lot of unlabeled data where the user did not explicitly indicate any activity. 
The tables in Figure 3 show the average amount of data (feature vectors) being collected for each class from each user and the average recording time. In the case of the "run" activity, for example, we collected about 7800 feature vectors (i.e., 13 minutes of recording) for each user. As already explained, each of these feature vectors consists of 9 sensor readings (3 3D accelerometer).

In the table we also report the average distance between feature vectors of the same class (inter-class distance) and average distance between feature vectors of one class and vectors of other classes (intra-class distance). The fact that inter-class distance is lower than intra-class distance support the use of IB algorithms for classification.

In order to illustrate the structure of our dataset we also executed multidimensional scaling [26] on our data (to represent 9-dimensions vectors in 2 dimensions). The result is depicted in Figure 4 and shows the clustered structure of the different classes.

The labels given to the recorded activities reflect what the user did at the time of data collection. In the activities dataset we selected the above 16 activities/labels with the goal of testing our system with an organized and heterogeneous set of actions. In the real-life dataset labels reflect what the user annotated for ground-truth recording.

The use of general labels such as: "dance" or "clean kitchen's racks" does not imply that we are able to reliably recognize every kind of "dancing" or "cleaning kitchen's racks" activities. We are aware that the dataset we collected both for training and testing purposes does not contain all the possible expressions of those general activities.

Our system produces the results discussed in Section 6, within the scope of the activities' expressions we recorded.

Nevertheless, the ability of effectively classifying specific activities can be combined with other approaches to allow the system to recognize more complex and general actions [27].

\section{Experimental Results}

We conducted several experiments to verify the accuracy of the algorithms proposed in Section 3 with our datasets. First, we compared IB1, IB3, IB6, DD3, DD6, CLOF3 and CLOF6 on the same dataset, using feature vectors described in Section 3.1. The number after the name of the 


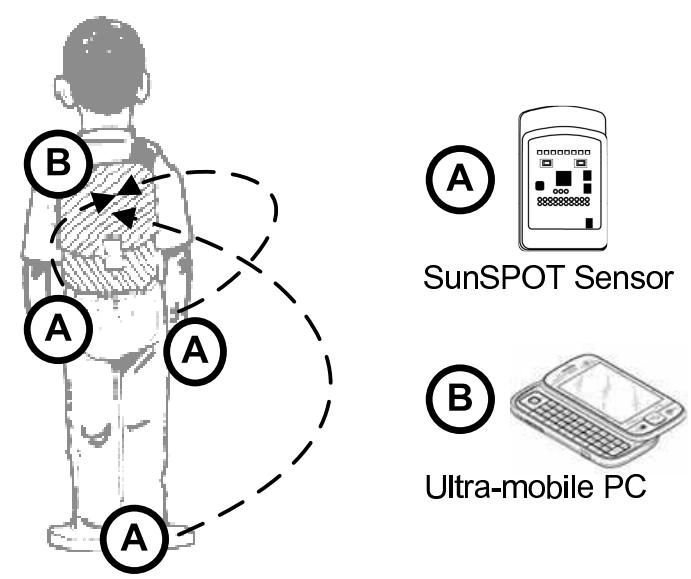

Figure 2: Data collection setup.

algorithm represents the number $k$ of searched neighbors. We tested the proposed algorithms only for values of $k<=6$, since we empirically found that higher values increase the computational complexity without increasing the accuracy of the classification.

To run the experiments we divided the datasets described in Fig. 3 among training and testing sets. In particular, in all the experiments we use 300 feature vectors for the testing set, and a training set varying from 30 to 240 feature vectors (as detailed in the following). On the basis of this set up, we adopt a cross validation approach by shifting the selection of the testing set across our data. This enables us to report results that are less dependent on the dataset. Furthermore, the final outcome is less sensitive to overfitting since the system is trained with different data "folds".

For all the experiment we computed precision and recall of the resulting classification. Thats is, for each class X:

$$
\begin{gathered}
\text { Precision }=\frac{\mid\{\text { vectors classified as } \mathrm{X}\} \cap\{\text { vectors that are actually } \mathrm{X}\} \mid}{\mid\{\text { vectors classified as } \mathrm{X}\} \mid} \\
\text { Recall }=\frac{\mid\{\text { vectors classified as } \mathrm{X}\} \cap\{\text { vectors that are actually } \mathrm{X}\} \mid}{\mid\{\text { vectors that are actually } \mathrm{X}\} \mid}
\end{gathered}
$$

Then we averaged results from different classes. It is worth noting that, since we have a testing set of 300 vectors for all the classes, we can just compute the average without any weighting. 


\begin{tabular}{|c|c|c|c|c|}
\hline Activity & Avg. \# vectors & Avg. rec. time & Avg. inter-class d. & Avg. intra-class d. \\
\hline stir pasta (cooking) & 3900 & $6 \mathrm{~min}$ & 0.59 & 1.67 \\
\hline climb stairs & 10800 & $18 \mathrm{~min}$ & 1.41 & 2.06 \\
\hline sleep (lie in bed) & 3700 & $6 \min$ & 0.02 & 3.39 \\
\hline run & 7800 & $13 \mathrm{~min}$ & 2.82 & 2.91 \\
\hline use pc & 15400 & $25 \mathrm{~min}$ & 0.51 & 2.31 \\
\hline wash hands & 1900 & $3 \min$ & 0.57 & 1.66 \\
\hline use ps3 & 1900 & $3 \mathrm{~min}$ & 0.17 & 1.89 \\
\hline read on the sofa & 1200 & $2 \min$ & 0.21 & 1.92 \\
\hline take the elevator & 2700 & $4 \mathrm{~min}$ & 0.35 & 2.15 \\
\hline ride a bike & 3500 & $6 \min$ & 0.87 & 1.69 \\
\hline stand still & 8800 & $14 \mathrm{~min}$ & 0.21 & 1.77 \\
\hline drive the car & 22500 & $37 \mathrm{~min}$ & 0.69 & 2.47 \\
\hline eat pasta & 4200 & $7 \mathrm{~min}$ & 0.47 & 1.97 \\
\hline drink standing still (bar) & 3900 & $6 \mathrm{~min}$ & 0.49 & 1.83 \\
\hline walk & 28100 & $47 \mathrm{~min}$ & 1.39 & 2.15 \\
\hline dance & 3300 & $5 \min$ & 1.48 & 2.01 \\
\hline Activity & Avg. \# Samples & Avg. rec. time & Avg. inter-class d. & Avg. intra-class d. \\
\hline chat being sat & 12600 & $21 \mathrm{~min}$ & 0.07 & 2.39 \\
\hline drink standing & 6000 & $10 \mathrm{~min}$ & 0.63 & 1.44 \\
\hline set up the table & 15600 & $26 \min$ & 0.86 & 1.43 \\
\hline clean kitchen racks & 3600 & $6 \min$ & 0.84 & 1.42 \\
\hline eat & 13800 & $23 \mathrm{~min}$ & 1.08 & 1.5 \\
\hline drive the car & 9600 & $16 \mathrm{~min}$ & 1.64 & 1.96 \\
\hline clean the table & 2400 & $4 \min$ & 1.26 & 1.86 \\
\hline chat standing & 26400 & $44 \mathrm{~min}$ & 0.79 & 1.46 \\
\hline work at the pc & 31800 & $53 \mathrm{~min}$ & 1.2 & 1.51 \\
\hline read on the sofa & 8400 & $14 \mathrm{~min}$ & 0.99 & 1.65 \\
\hline wash hands & 1200 & $2 \min$ & 0.74 & 1.41 \\
\hline take s. from fridge & 1800 & $3 \min$ & 0.75 & 1.48 \\
\hline
\end{tabular}

Figure 3: (top) Activity dataset. (bottom) Real-life dataset

All the experiments except those in Section 6.3 have been conducted by using, for both training and testing, data coming from the same user. Thus, the measured precision and recall in all the experiments refers to this case. More in detail, we set up the experiment so that during cross validation, the 300 -vector testing set is always associated with a training set coming from the same user.

Figure 5 shows the precision and recall levels reached with an increasing number of training instances for the various classifiers. It is interesting to note that even a small number of training instances per class (approximately 50 ) is enough to reach high precision and recall. Moreover, it is clear from the graph that IBk and DDk algorithms outperform CLOFk. Considering the fact that these algorithms may run on resource constrained devices (see Section 7), IB1 is clearly the best choice. In fact, even though its performance is similar to IB3, IB6 and DDk, it is much simpler and, therefore, faster to be executed. For this reason, we used IB1 in all the experiments reported in the following of this section.

It is worth mentioning that, without any kind of optimizations, by simply applying IB1 to a training set composed of only 200 instances of 9 attributes (3 axis, 3 accelerometers) it is possible to achieve remarkable precision and 


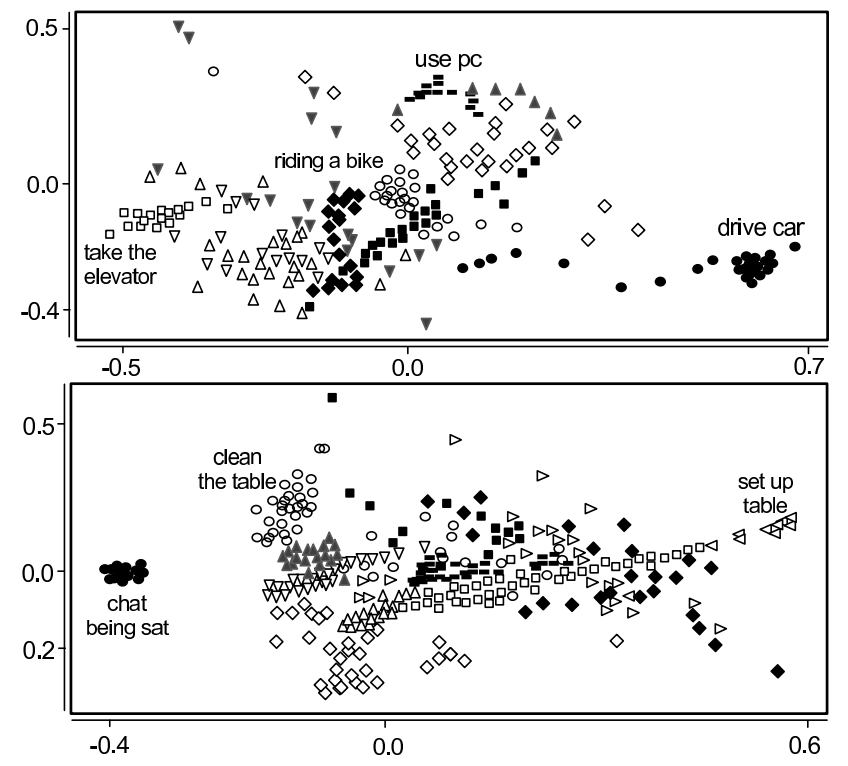

Figure 4: (top) Activity dataset. (bottom) Real-life dataset

recall levels. In particular, about $85 \%$ on the activity dataset while about $75 \%$ on the real life data set. This experiment supports the contribution claims made at the beginning of the paper: the simple IB1 algorithm can classify accelerometer data with about $80 \%$ precision and recall, with a limited number of training examples. This fact also supports the idea of using IB algorithms on resource-constrained sensors (see Section 7).

Although a fair comparison with the state-of-the-art reported in Section 2 would require using the same dataset, the obtained results indicate that - at least with regard of the set of action we considered - instance-based algorithms exhibit performance in line with the current best practices.

We were also interested in discovering whether the information contributions coming from the three sensors were equal or not. Thus we applied IB1 to the same dataset used in the experiments above but modified to suppress the contribution of one or more sensors. In Figure 6 we plotted precision and recall reached by IB1, in the activity dataset, increasing the number of training instances, using one sensor, two sensors, all three sensors, and each of them alone. The plot shows that the sensors applied to the arm (S1) and to the leg (S2) provide a similar amount of information, while the one applied to the waist (S3) is $10 \%$ less effective. In summary, even though the waist-applied sensor gives a slightly smaller contribution, all three sensors 
a)

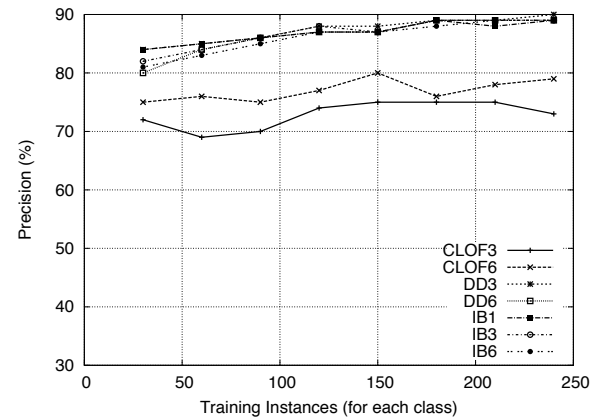

c)

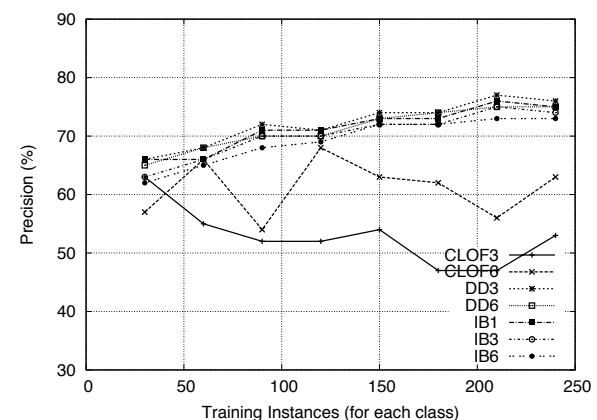

b)

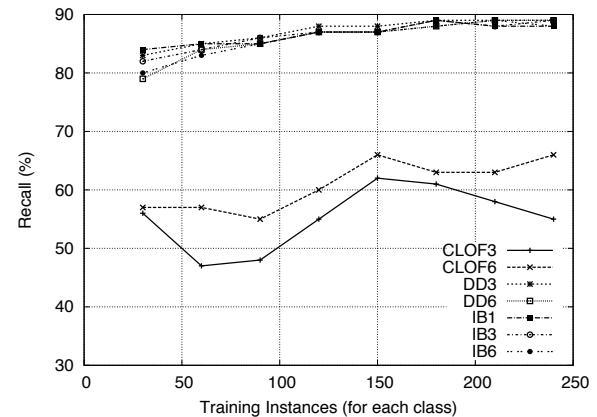

d)

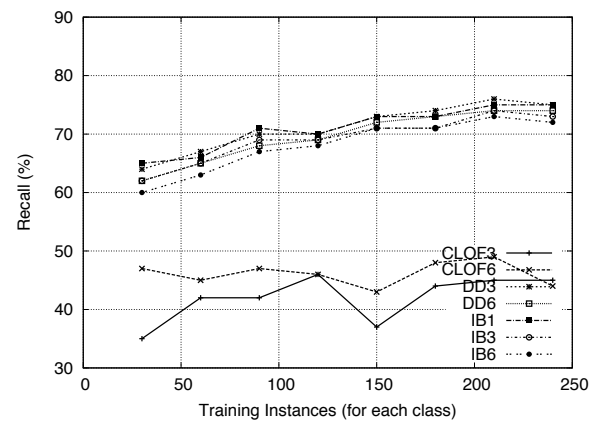

Figure 5: Precision and recall levels achieved by several algorithms (IBk, DDk, CLOFk) using an increasing number of training instances (a,b activity dataset, $\mathbf{c}, \mathbf{d}$ real-life dataset).

are needed to reach an overall precision and recall both close to $90 \%$ in the activity dataset, and to $75 \%$ in the real life dataset.

\subsection{Dealing with Time}

We tried to investigate if precision and recall levels of IB1 could increase using different feature vectors. In particular we tried to introduce the time variable in our classification problem by "embedding temporal information in the feature vectors" in different ways (see Section 4.1). Figure 7(a) shows precision and recall of IB1 using an increasing number of training instances each one composed of $n$ samples (9 attributes each) concatenated over a sliding window $(n=10,20)$. In Figure $7(\mathrm{~b})$ the feature vector is composed of a single sampling (9 attributes) followed by mean and variance of each attribute (18 attributes) over a sliding window of the previous $n$ elements $(n=10,20)$. In Figure $7(\mathrm{c})$ the feature vector is composed by a single sampling (9 attributes) followed by mean and variance of the magnitude of 
a)

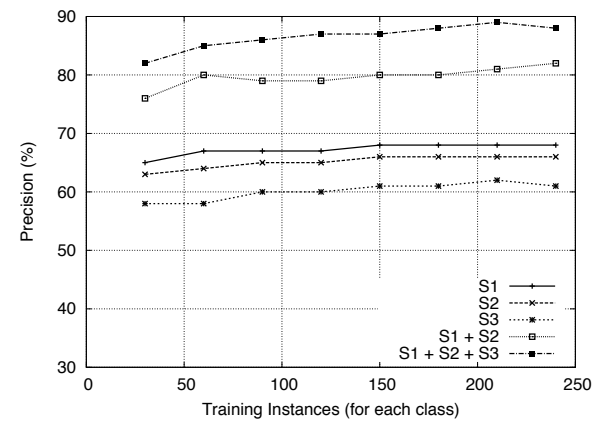

c)

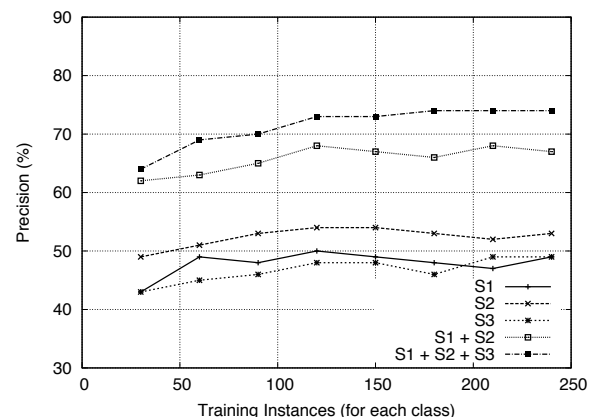

b)

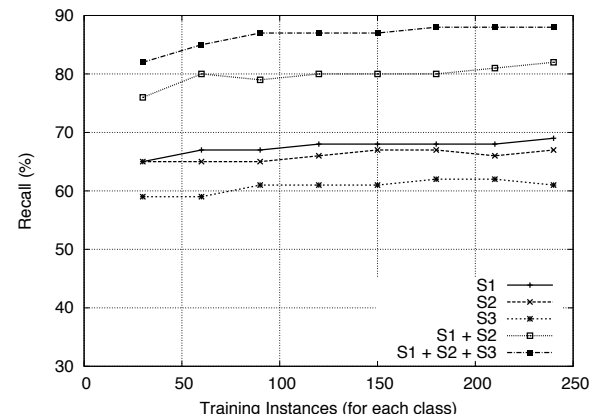

d)

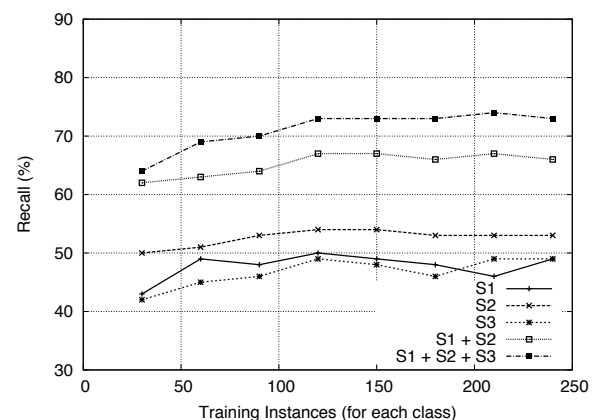

Figure 6: The information contribution of each sensor. The plot shows that the sensors applied to the arm (S1) and to the leg (S2) contribute with a similar amount of information, while the one applied to the waist (S3) is approximately $10 \%$ less informative (a,b activity dataset, $\mathbf{c}, \mathbf{d}$ real-life dataset).

each sensor (6 attributes) over a sliding window of the previous $n$ elements $(n=10,20)$.

These simple temporal representations are effective and improve classification precision and recall by more than $10 \%$. Considering the fact that all three variations produce similar results, the simplest one has to be preferred. Due to this, the most valuable option is the third one. In fact, its precision and recall levels are only slightly worse than the other two (3-5\%) but the sample vector is composed of only 15 attributes instead of of 27(b) or $90 / 180(a)$.

Finally, Figure 7(d) shows the results achieved using "majority voting over temporal windows" optimization (see Section 4.1). We divided the dataset in windows with a variable length. After the classification process, each window was associated with its most frequent label. This technique is effective and precision and recall close to $100 \%$ can be achieved. With regard to this result, 
a)

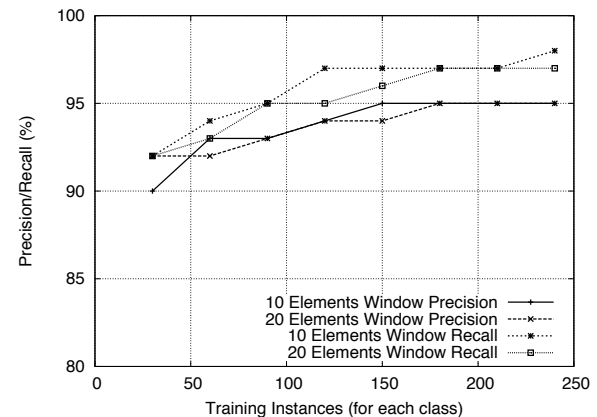

c)

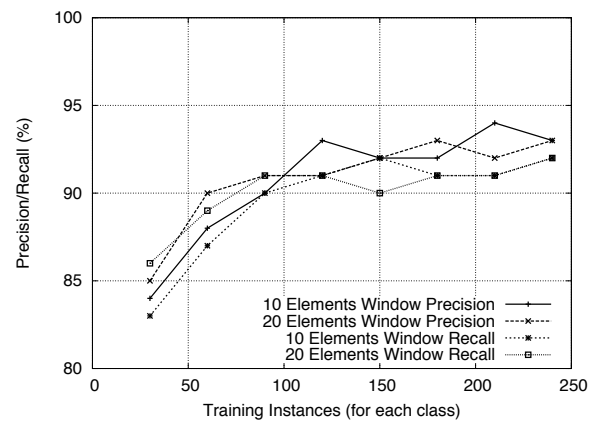

b)

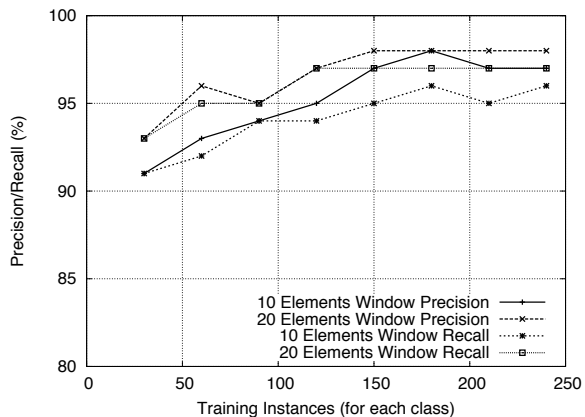

d)

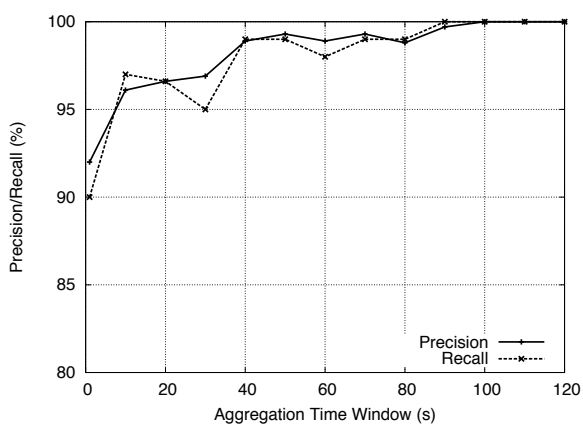

Figure 7: Activity dataset. Graph showing precision and recall against number of training instances, using the temporal optimization of Section 4.1. Training instances composed of: (a) $n$ samples (9 attributes each) concatenated over a sliding window $(n=10,20)$. (b) $n$ samples (9 attributes each), mean and variance of each attribute (18 attributes) over a sliding window of $n$ elements $(n=10,20)$. (c) $n$ samples (9 attributes each), mean and variance of each sensor (6 attributes) over a sliding window of $n$ elements $(n=10,20)$. (d) Graph showing the effect of temporal aggregation on the overall precision and recall. Classified instances are aggregated over increasing time windows (x-axis). For each window the most frequent ground truth and the most frequent classification are accounted.

it is again worth noticing that this experiment has been conducted using cross validation. This indicates that the reported result is less dependent on the dataset and that it is eventually less sensitive to the problem of overfitting since it derives form training with different data.

Figure 8 illustrates the results of the above experiment conducted over the real-life data set, and in this case also similar conclusions can be reached.

\subsection{Dealing with New Classes}

To test the effectiveness of the proposed approach to recognize new activities, described in Section, we performed the following set of experiments. 
a)

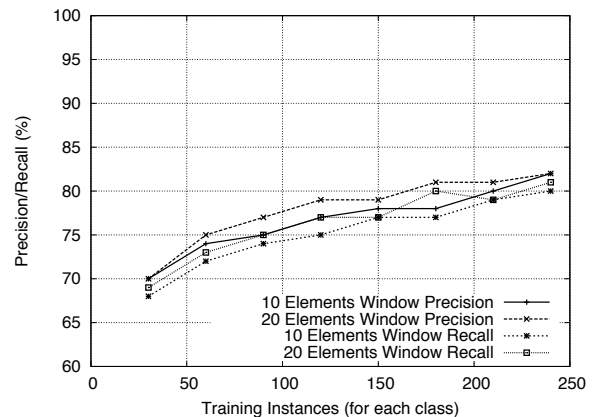

c)

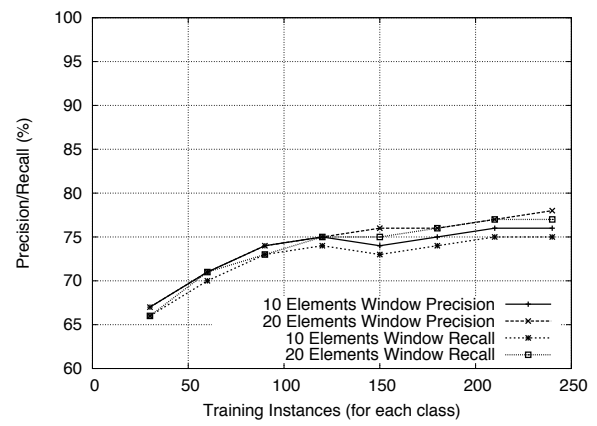

b)

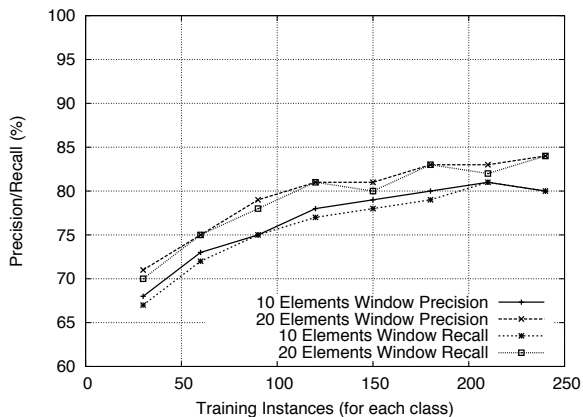

d)

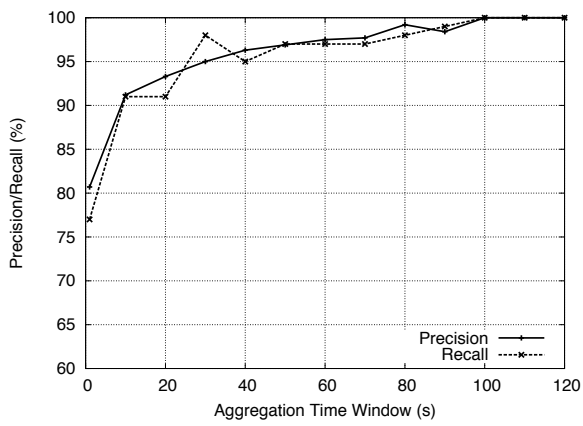

Figure 8: Real-life dataset. Graph showing precision and recall against number of training instances , using the temporal optimization of Section 4.1. Training instances composed of: (a) $n$ samples (9 attributes each) concatenated over a sliding window $(n=10,20)$. (b) $n$ samples (9 attributes each), mean and variance of each attribute (18 attributes) over a sliding window of $n$ elements $(n=10,20)$. (c) $n$ samples (9 attributes each), mean and variance of each sensor (6 attributes) over a sliding window of $n$ elements $(n=10,20)$. (d) Classified instances are aggregated over increasing time windows (x-axis). For each window the most frequent ground truth and the most frequent classification are accounted.

We conducted a separate test for each of the 16 classes in our activity dataset. For each class we created a training set composed of 250 vectors from each class but the selected one (i.e., $15 \cdot 250$ vectors). Moreover, we created a test set containing 250 vectors of all the classes (i.e., 16 - 250 vectors).

These vectors have been chosen form the dataset in Fig. 3 and then permutated to engage a cross validation schema.

The idea is to investigate how the algorithm deals with the class that is in the testing set, but not in the training set. We ran the LCF algorithm to discover which samples in the testing set were not present in the training set. In particular, we annotated how many of the instances of the missing class have been correctly classified as new. 


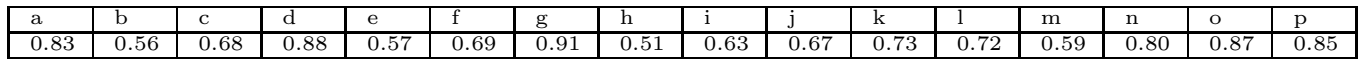

Figure 9: Table describing the precision of LCF in new events classification. Actions used are: (a) stand still; (b) sleep (lie in bed); (c) read sat on the sofa; (d) walk; (e) run; (f) drive the car; (g) climb stairs; (h) use pc (type and move the mouse); (i) use playstation 3 (sat with the joypad in hand); (j) stir pasta (while cooking); (k) eat pasta; (l) drink at the bar (drink standing still); (m) dance (step feet and move hands rhythmically); (n) ride a bike; (o) take the elevator; (p) wash hands;

Figure 9 presents the results. It shows the precision of the algorithm in identifying the new class. The table shows for example that if the action (a) stand still is not in the training set, then the LCF algorithm correctly classifies it as new in $83 \%$ of the times. In the remaining $17 \%$ of the times, the action is incorrectly classified as one of the other activities.

The result shows that, for the most of the actions, more than $50 \%$ of the instances were correctly classified as new. However it is worth mentioning that these results have a considerable variance and depend on the number and type of the classes present in the training set. For example, instances belonging the dancing class are frequently misclassified as running instead of new because of the similarities between these two actions. Obviously, if the class running was not present in the training set, the number of errors would be greatly reduced.

In this experiments, we empirically set $k=6, w=0.5$ and $t h=4.0$ by cycling through all the possible values of these parameters to find the best result. To be more specific we explored $0<k<10,0<w<1$ and $0<t h<10$ using a discrete step of 0.1 . We are aware that this methodology is prone to overfitting and that this result has been biased by the data we used. However, considering the early stage of this part of our work, we aim simply to show that instance-based algorithms can be a suitable tool for recognizing novel classes.

Similar approaches, grounded on statistical significance tests, have been presented in $[24,25]$. The use of statistical tests rather than a single threshold can improve the robustness and the correctness of the approach. In $[24,25]$ authors report $90 \%$ precision in identifying new (NULL) classes. In our future work we will apply statistical significance tests to improve our results.

\subsection{Moving the Training Set}

A desirable property for every event classification system is to be resistant to overfitting. This property makes a system robust to the "background 
noise" during the classification and may greatly simplify the deployment and eventual updates. While a system prone to overfitting has to be tuned for every user or usage, a less sensitive one can share a common training set among different installations. In addition, sharing samples among users would speed up the training phase of the algorithm. For example, a sport application could be provided with a pre-collected set of labeled samples to classify sport activities. A user of this application could try to classify his/her own activities on the basis of the predefined samples, thus zeroing the bootstrap training phase.

While in all the previous experiments the training and the testing sets were recorded by the same user, in this experiment the training and the test sets were recorded by two different users.

We tested two kinds of feature vectors for this experiment: (i) vectors composed by a single sampling (9 attributes) and mean and variance of the magnitude of each sensor ( 6 attributes) over a sliding window of $n$ elements $(n=10,20)$. (ii) The same kind of vectors, but normalized to a fixed range, in order to reduce differences across users. Figure 10 shows the precision and recall levels against the number of training instances. Unfortunately the graphs show a robust decrease (40\%-55\% against 90\%) in precision and recall suggesting a limited portability of training sets over different installations. This is due to the inherent idiosyncrasies of each user's movements that make the sharing of samples rather inaccurate.

To better illustrate this problem we analyzed the confusion matrix associated with this classification (see Figure 11). Large errors are mainly associated to rather similar activities (e.g., looking at the first row, the "drink at the bar" activity is often misclassified into "stir pasta". Similarly "eat pasta" is often confused with "stir pasta").

The main problem here relates to our use of labels to describe activities. As discussed in Section 5, our dataset is rather limited: data representing the "drink at the bar" activity, for example, does not cover all the possible expressions of such an activity, so different users might label "drink at the bar" with some rather different movements. To overcome this limitation, on the one hand a larger dataset comprising more expressions of the same activities should be recorded. On the other hand, novel features should be extracted from the sensor data. In particular, the spectral characteristics of the data seem to be a good candidate for this kind of application [7]. In our future work we will combine spectral characteristics in the feature vectors so as to allow for training set portability across users. 
a)

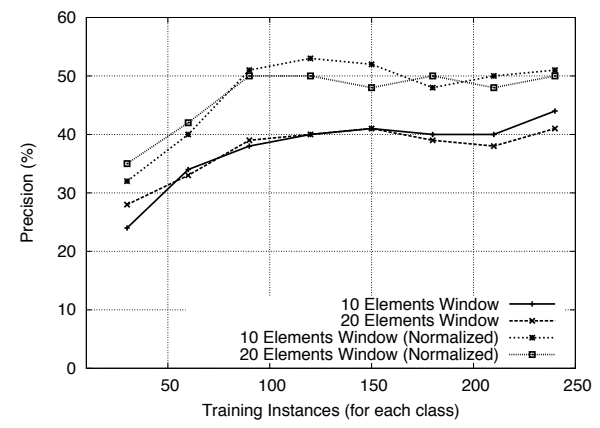

b)

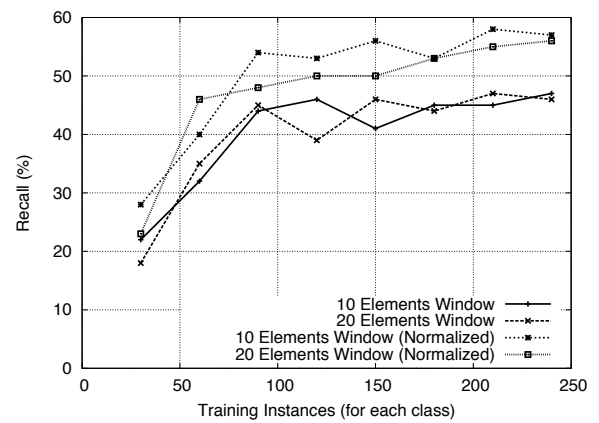

Figure 10: Graph showing precision and recall against number of training instances. In this case the feature vector is composed by a single sampling (9 attributes) and mean and variance of the magnitude of each sensor ( 6 attributes) over a sliding window (10/20 items). The training and test set are composed by the same actions performed by two different users. This shows a limited portability of training sets over different installations. Due to this, it is important to tune the classification system on each user.

\section{Running Instance-based Algorithms on Resource-constrained Sensors}

Instance-based classification algorithms are usually known to have two main performance drawbacks: (i) high classification time, and (ii) large storage space needed. In this section we illustrate that none of these concerns apply to this application scenario. We show this both with simple calculations and with some experiments on different sensor platforms.

\subsection{Classification Time}

Instance-based classification typically has classification times that are much larger than other classifiers. For example, while with a linear classifier only a dot product needs to be computed to classify a test sample (to find out the closest class vector), instance-based algorithms require the training set to be ranked for similarity with the test sample, which is much more expensive. This is a characteristic of "lazy" learning methods, since they do not have a true training phase and thus defer all the computation at classification time. The fundamental operation that needs to be performed by these algorithms is to identify the $\mathrm{k}$ training samples closest to the sample to be classified. While exhaustive search requires $\mathrm{O}(\mathrm{N})$ operations, where $\mathrm{N}$ is the number of samples in the training set, there exist advanced data 


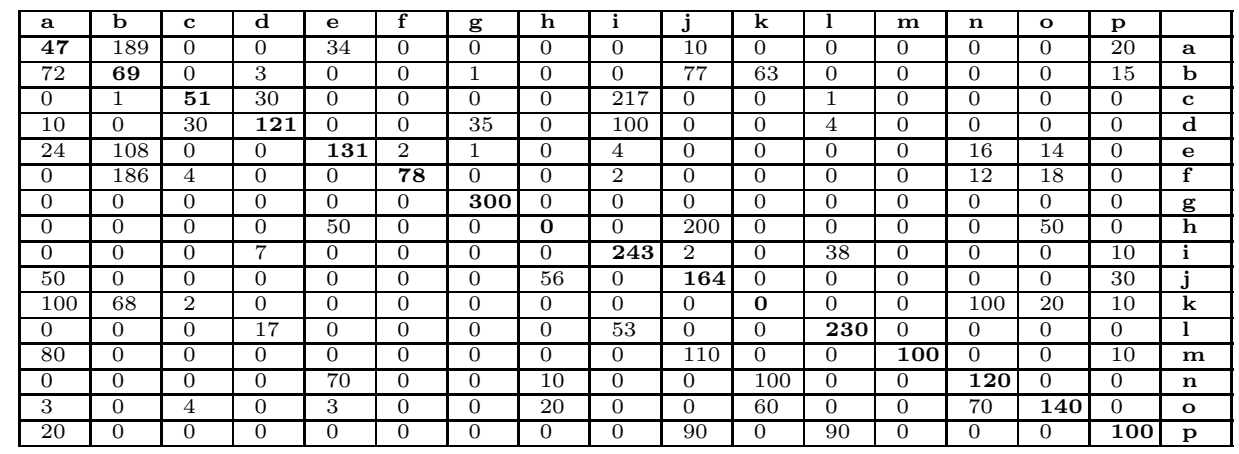

Figure 11: Confusion matrix for experiment of Figure 10. First row is classification. Last Column groundtruth. $\mathrm{a}=$ drink at the bar (drink standing still); $\mathrm{b}=$ stir pasta (while cooking); $\mathrm{c}=$ ride a bike; $\mathrm{d}=$ dance ( the car; $\mathrm{f}=$ eat pasta; $\mathrm{g}=\mathrm{run} ; \mathrm{h}=$ sleep (lie in bed); $\mathrm{i}=$ climb stairs; $\mathrm{j}=$ stand still; $\mathrm{k}$ $=$ use pc (type and move the mouse); $\mathrm{l}=$ walk; $\mathrm{m}=$ wash hands; $\mathrm{n}=$ use playstation 3 (sat with joypad in hand); o = read on the sofa; $\mathrm{p}=$ take the elevator

structures (kd-trees) [28] able to identify the k nearest neighbors with $\mathrm{O}(\mathrm{log}$ N) operations.

To assess the feasibility of our proposal, also in the simple case of exhaustive - $\mathrm{O}(\mathrm{N})$ - search, we assumed a scenario with 100 activities to be recognized and 100 training samples used to describe each of them. In particular, we focus on the case illustrated in Figure 7(c). In this case, acceleration data are sampled at $10 \mathrm{~Hz}$ by three sensors. Sensors sample data and send them, using a wired or wireless connection, to the main sensor node (in charge of performing the IB classification). The main node receives all the data and computes means and variances over a window of 10 elements $(1 \mathrm{~s})$ to get the feature vector. Assuming that human activities last for a minimum of $1 \mathrm{~s}$, the main sensor node can output class labels at $1 \mathrm{~Hz}$, so it has to classify one feature vector composed by 15 floats every second. We considered the time needed to receive and average the 10 samples in the window as negligible.

Although in our setup we used an UMPC to run classification, in this experiment we wanted to test the computational burden of IB classification on a wireless sensor. Given these assumptions, we measured the time required by both Sun SPOT and MicaZ sensors (http://www.xbow.com) to compute the Euclidean distance between two 15-float samples. We implemented a simple algorithm in Java (Sun SPOT) and NesC (MicaZ) and ran it 1000 times on both the devices. On average it took approximately $0.01 \mathrm{~ms}$ on a Sun SPOT sensor and $0.05 \mathrm{~ms}$ on a MicaZ sensor (see Figure 12). 


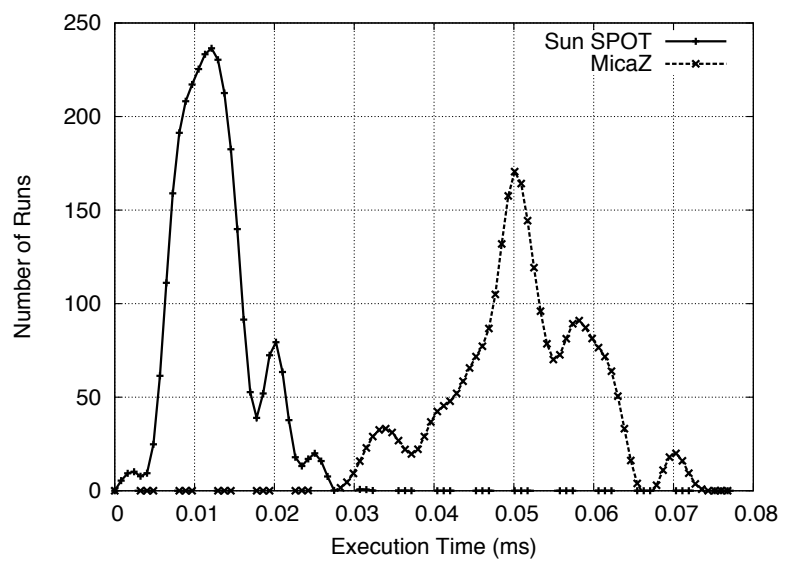

Figure 12: Distribution of time to compute distances among feature vectors in Sun SPOT and MicaZ sensor platforms.

Considering 100 actions, each of them described by 100 samples, and a simple exhaustive search over the whole dataset composed in this case by 10000 training samples, we can estimate an overall execution time of $0.1 \mathrm{~s}$ on Sun SPOT sensors and $0.5 \mathrm{~s}$ on MicaZ sensors. It is worth noting that this search has to be done only once every second. Moreover, by exploiting efficient data structures able to avoid exhaustive searches like kd-trees, the overall execution time would be in the order of $1 \mathrm{~ms}$ on both the sensors.

\subsection{Memory Requirements}

Instance-based classification algorithms tend to require more memory than other classifiers. While most classifiers (e.g., neural networks and Bayesian networks) construct an explicit and declarative representation of the class of interest, instance-based algorithms simply store all the samples in the training set. So, while the class representation can be compact (e.g., a linear classifier only requires 1 vector per class), the storage of the training samples is much larger.

The above two drawbacks are almost negligible in our scenario. We verified (see Figure 7 and 8) that state-of-the-art classification performances can be achieved by storing about 100 feature vectors per class and that no significant improvement can be expected by increasing that number.

For example, considering the case of Figure 7(c) in which we have samples of 15 floats: single sensor data ( 9 floats) followed by mean and variance of the magnitude of each sensor over a sliding window ( 6 floats). Assuming 4 bytes 
per float, then we have 60 bytes per feature vector, and thus $6 \mathrm{~KB}$ per class. In our test-bed, each sensor is a Sun SPOT provided with 512KB RAM and 4MB Flash memory. Thus, by storing the feature vectors in the node flash memory, we can theoretically recognize about 650 activities. Considering a testbed of MicaZ sensor node, each having 512KB Flash memory, we would still be able to represent 85 activities.

\section{Conclusions and Future Work}

In this paper we described the use of instance-based algorithms to infer user's activities on the basis of accelerometer data acquired from body-worn sensors. In particular, we highlighted that instance-based algorithms provide state-of-the-art performance, are simple to implement, and can run on resource-constrained sensor platforms. Furthermore we showed that they can achieve remarkable precision and accuracy levels in classifying simple and specific activities. As a consequence, this approach is a promising starting point for building next-generation systems able to recognize more complex activities [27].

Despite these encouraging results, further work is needed to improve the portability of training sets across individuals and to better identify new classes at run time. In addition, larger-scale experiments may be needed to enable the development of practical tools for the automatic recognition of user activities.

\section{References}

[1] M. Hanson, H. Powell, A. Barth, K. Ringgenberg, B. Calhoun, J. Aylor, J. Lach, Body area sensor networks: Challenges and opportunities, IEEE Computer 42 (1) (2009) 58-65.

[2] A. Campbell, S. Eisenman, N. Lane, E. Miluzzo, R. Peterson, H. Lu, X. Zheng, M. Musolesi, K. Fodor, G. Ahn, The rise of people-centric sensing, IEEE Internet Computing 12 (4) (2008) 12-21.

[3] G. Roussos, V. Kostakos, Rfid in pervasive computing: State-of-theart and outlook, Elsevier Pervasive and Mobile Computing 5 (1) (2009) 110-131. 
[4] D. Patterson, L. Liao, D. Fox, H. Kautz, Inferring high-level behavior from low-level sensors, in: International Conference on Ubiquitous Computing, ACM, Seattle (WA), USA, 2003, pp. 73-89.

[5] T. Mitchell, Machine Learning, McGraw Hill, New York (NY), USA, 1996.

[6] T. Huynh, M. Fritz, B. Schiele, Discovery of activity patterns using topic models, in: International conference on Ubiquitous computing, ACM, Seoul, Korea, 2008, pp. 10-19.

[7] T. Choudhury, S. Consolvo, B. Harrison, J. Hightower, A. LaMarca, L. LeGrand, A. Rahimi, A. Rea, G. Bordello, B. Hemingway, P. Klasnja, K. Koscher, J. Landay, J. Lester, D. Wyatt, D. Haehnel, The mobile sensing platform: An embedded activity recognition system, IEEE Pervasive Computing 7 (2) (2008) 32-41.

[8] H. Junker, O. Amft, P. Lukowicz, G. Tröster, Gesture spotting with body-worn inertial sensors to detect user activities, Elsevier Pattern Recognition 41 (6) (2008) 2010-2024.

[9] E. Miluzzo, N. D. Lane, K. Fodor, R. Peterson, H. Lu, M. Musolesi, S. B. Eisenman, X. Zheng, A. T. Campbell, Sensing meets mobile social networks: the design, implementation and evaluation of the cenceme application, in: Conference on Embedded Networked Sensor Systems, ACM, Raleigh (NC), USA, 2008, pp. 337-350.

[10] L. Bao, S. Intille, Activity recognition from user-annotated acceleration data, in: International Conference on Pervasive Computing, Springer, Linz, Austria, 2004, pp. 1-17.

[11] A. Torralba, R. Fergus, W. T. Freeman, 80 million tiny images: a large dataset for non-parametric object and scene recognition, IEEE Transactions on Pattern Analysis and Machine Intelligence 30 (11) (2008) 1958-1970.

[12] C. Plant, C. Bohm, B. Tilg, C. Baumgarten, Enhancing instance-based classification with local density: a new algorithm for classifying unbalanced biomedical data, Oxford Bioinformatics 22 (8) (2006) 981-988. 
[13] F. Sebastiani, Machine learning in automated text categorization, ACM Computing Surveys 34 (1) (2002) 1-47.

[14] S. Lee, K. Mase, Activity and location recognition using wearable sensors, IEEE Pervasive Computing 1 (3) (2002) 24-32.

[15] J. Mantyjarvi, J. Himberg, T. Seppanen, Recognizing human motion with multiple acceleration sensors, in: International Conference on Systems, Man, and Cybernetics, Vol. 2, IEEE, Tucson (AZ), USA, 2001, pp. $747-752$.

[16] F. Foerster, M. Smeja, J. Fahrenberg, Detection of posture and motion by accelerometry: a validation in ambulatory monitoring, Elsevier Computers in Human Behavior 15 (1999) 571-583.

[17] K. Aminian, P. Robert, E. Buchser, B. Rutschmann, D. Hayoz, M. Depairon, Physical activity monitoring based on accelerometry: validation and comparison with video observation, Springer Medical and Biological Engineering and Computing 37 (3) (1999) 304-328.

[18] N. Kern, B. Schiele, A. Schmidt, Multi-sensor activity context detection for wearable computing, in: European Symposium on Ambient Intelligence, Veldhoven, The Netherlands, 2003, pp. 220-232.

[19] G. Chambers, S. Venkatesh, G. West, H. Bui, Hierarchical recognition of intentional human gestures for sports video annotation, in: International Conference on Pattern Recognition, IEEE, Quebec City, Canada, 2002, pp. 1082-1085.

[20] C. Randell, H. Muller, Context awareness by analysing accelerometer data, in: International Symposium on Wearable Computers, IEEE, Atlanta (GE), USA, 2000, pp. 175-176.

[21] K. V. Laerhoven, O. Cakmakci, What shall we teach our pants?, in: International Symposium on Wearable Computers, IEEE, Atlanta (GE), USA, 2000, p. 77.

[22] N. Ravi, N. Dandekar, P. Mysore, M. Littman, Activity recognition from accelerometer data, in: Innovative Applications of Artificial Intelligence, AAAI Press, Pittsburgh(PA), USA, 2005, pp. 1541-1546. 
[23] N. Bicocchi, M. Lasagni, M. Mamei, A. Prati, R. Cucchiara, F. Zambonelli, Pervasive self-learning with multi-modal distributed sensors, in: PerAda Workshop, Conference on Self-organizing and Self-adaptive Systems, IEEE, Venice, Italy, 2008, pp. 61-66.

[24] D. Barbara, C. Domeniconi, J. Rogers, Detecting outliers using transduction and statistical testing, in: International Conference on Knowledge Discovery and Data Mining, ACM, Philadelphia (PA), USA, 2006, pp. 55-64.

[25] C. Park, H. Shim, On detecting an emerging class, in: International Conference on Granular Computing, IEEE, San Jose (CA), USA, 2007, pp. 265-270.

[26] U. Brandes, C. Pich, Eigensolver methods for progressive multidimensional scaling of large data, in: International Symposium on Graph Drawing, Karlsruhe, Germany, 2006, pp. 42-53.

[27] E. Kim, S. Helal, D. Cook, Human activity recognition and pattern discovery, IEEE Pervasive Computing 9 (1) (2010) 48 -53.

[28] J. Predd, S. Kulkarni, H. Poor, Distributed learning in wireless sensor networks, IEEE Signal Processing 23 (4) (2006) 56 - 69. 